\title{
Étude des équilibres des phases dans le système Ag-Sn-In à $280^{\circ} \mathrm{C}$
}

\author{
E.S. Dobrev ${ }^{1,2}$, G.P. Vassilev ${ }^{1}$ et J.-C. Tédenac ${ }^{2}$ \\ ${ }^{1}$ Faculté de Chimie, Université de Sofia, 1 James Bourchier Av., 1164 Sofia, Bulgarie \\ ${ }^{2}$ L.P.M.C., Université Montpellier II, PI. Eugène Bataillon, 34095 Montpellier, France
}

\begin{abstract}
The Ag-Sn system is among the most studied ones in the prospective of the abandon of the lead containing alloys. On the other hand the temperatures of these alloys are higher than those of the now used $\mathrm{Pb}-\mathrm{Sn}$ eutectic alloys. Consecutively the addition of $\mathrm{In}-\mathrm{a}$ low melting element is interesting from the new materials' development. The isothermal section of the $\mathrm{Ag}$-Sn-In system at $280^{\circ} \mathrm{C}$ was studied by using optical and scanning electron (SEM) microscopies, X-ray diffraction, differential scanning calorimetry (DSC) and Vickers' microhardness measurements. The microhardness values of $\mathrm{D}(\mathrm{Ag}), \mathrm{H}\left(\mathrm{Ag}_{4} \mathrm{Sn}\right)$ and ] phases were measured. The ternary eutectic's position reported in the literature was confirmed. The respective third element's solubilities were determined.
\end{abstract}

\begin{abstract}
Résumé. Le système Ag-Sn est parmi les systèmes les plus étudiés dans la perspective de l'abandon des alliages à base de $\mathrm{Pb}$. D'autre part les températures de fusion de ces alliages sont plus élevées que celle de l'eutectique $\mathrm{Pb}-\mathrm{Sn}$ actuellement employé. Consécutivement, l'addition d'indium, un élément ayant température de fusion relativement basse, est un moyen intéressant pour la mise au point de nouvelles brasures. La coupe isotherme du système $\mathrm{Ag}$-Sn-In à $280^{\circ} \mathrm{C}$ a été construite à l'aide des microscopies optique et électronique à balayage (MEB), de la diffraction des rayons $\mathrm{X}$, de mesures de microdureté Vickers et de calorimétrie différentielle à balayage (DSC). Les microduretés des phases $\left.\mathrm{D}(\mathrm{Ag}), \mathrm{HAg}_{4} \mathrm{Sn}\right)$ et ] ont été mesurées. La position de l'eutectique ternaire rapportée antérieurement dans la littérature a été confirmée. Les solubilités du troisième élément respectif ont été déterminées.
\end{abstract}

\section{INTRODUCTION}

La nouvelle réglementation, concernant la préservation de l'environnement exige le remplacement des alliages à base de plomb, principalement employés dans l'industrie électronique. Le système Ag-Sn est parmi ceux les plus intéressants pour la mise au point de nouveaux matériaux. Certains alliages à base du système Ag-Sn sont déjà utilisés comme alliages de brasure, mais seulement pour applications spécialisées, exigeant des températures de fusion du contact supérieures à $183^{\circ} \mathrm{C}$ (la température de fusion de l'eutectique $\mathrm{Pb}-\mathrm{Sn}$ ). En même temps ils montrent des caractéristiques équivalentes ou supérieures à celles des alliages $\mathrm{Pb}$-Sn actuellement utilisés. Par conséquent, l'addition d'indium, un élément ayant une température de fusion relativement basse, est un moyen intéressant pour la mise au point de nouvelles brasures.

Les systèmes binaires limitrophes ont fait l'objet de nombreuses études expérimentales et optimisations thermodynamiques [1-12]. La compilation des données cristallographiques pour les phases binaires est présentée dans le Tableau 1.

Dans les systèmes Ag-In et Ag-Sn existent deux couples de phases isomorphes - les deux solutions solides à base d'argent et les phases ] $\left(\mathrm{Ag}_{4} \mathrm{Sn}\right.$ et $\left.\mathrm{Ag}_{3} \mathrm{In}\right)$. Par conséquent, la formation de solutions solides continues peut être attendue. 
Le but de ce travail est de vérifier les équilibres de phases dans le système Ag-Sn-In et d'obtenir des résultats plus détaillés sur les intervalles d'homogénéités des phases.

Tableau 1. Description des phases binaires pertinentes dans le système Ag-Sn-In.

\begin{tabular}{|c|c|c|c|c|c|}
\hline Phase & Intervalle & $\begin{array}{l}\text { Pearson } \\
\text { symbole }\end{array}$ & Groupe spatial & $\begin{array}{l}\text { Strukturbericht } \\
\text { désignation }\end{array}$ & Prototype \\
\hline $\mathrm{D}(\mathrm{Ag})$ & $\begin{array}{l}0-21 \text { at. } \% \mathrm{In} \\
0-\quad \text { at. } \% \mathrm{Sn}\end{array}$ & $\mathrm{cF} 4$ & $F m \overline{3} m$ & A1 & $\mathrm{Cu}$ \\
\hline $\mathrm{E}\left(\mathrm{In}_{3} \mathrm{Sn}\right)$ & $25-30$ at. $\%$ In & $\mathrm{cP} 2$ & $P m \overline{3} m$ & B2 & $\mathrm{CsCl}$ \\
\hline$]\left(\mathrm{Ag}_{3} \mathrm{In}\right)$ & $25-46$ at. $\%$ In & $\mathrm{hP2}$ & $P 6_{3} / m m c$ & A3 & $\mathrm{Mg}$ \\
\hline $\mathrm{D}^{\prime}\left(\mathrm{Ag}_{3} \mathrm{In}\right)^{* *}$ & 25 at. $\%$ In & $\mathrm{cP}^{*}$ & $P m \overline{3} m$ & $\ldots$ & $\ldots$ \\
\hline $\mathrm{J}\left(\mathrm{Ag}_{9} \mathrm{In}_{4}\right)$ & $31-33.5$ at. $\%$ In & cP52 & $P \overline{4} 3 m$ & $\mathrm{D} 8_{3}$ & $\mathrm{JCu}_{5} \mathrm{Zn}_{8}$ \\
\hline$M\left(\operatorname{AgIn}_{2}\right)^{* *}$ & 66.7 at. $\%$ In & $\mathrm{tI} 12$ & $I 4 / \mathrm{mcm}$ & $\mathrm{C} 16$ & $\mathrm{CuAl}_{2}$ \\
\hline Tetr. (In) & $0-11$ at.\% Sn & $\mathrm{t} \mathrm{t} 2$ & $I 4 / \mathrm{mmm}$ & A6 & In \\
\hline $\mathrm{E}_{1}^{* *}$ & $12-44$ at. $\% \mathrm{Sn}$ & tI2 & $14 / \mathrm{mmm}$ & A6 & In \\
\hline $\mathrm{J}_{1}^{* *}$ & $72-?$ at. $\% \mathrm{Sn}$ & $\ldots$ & $\ldots$ & $\ldots$ & $\ldots$ \\
\hline$(\mathrm{ESn})$ & $96.7-100$ at.\% Sn & tI4 & I4 ${ }_{1} /$ amd & A5 & $\mathrm{ESn}$ \\
\hline (DSn) & 100 & $\mathrm{cF} 8$ & $F d \overline{3} m$ & A4 & C-diamond \\
\hline $\mathrm{H}\left(\mathrm{Ag}_{3} \mathrm{Sn}\right)$ & $24-25$ at. $\% \mathrm{Sn}$ & oP8 & Pmmm & $\mathrm{D} 0_{\mathrm{a}}$ & $\mathrm{ECu}_{3} \mathrm{Ti}$ \\
\hline$]\left(\mathrm{Ag}_{4} \mathrm{Sn}\right)$ & $\sim 13-21.5$ at. $\% \mathrm{Sn}$ & $\mathrm{hP} 2$ & $\mathrm{~Pb}_{3} / m m c$ & A3 & $\mathrm{Mg}$ \\
\hline
\end{tabular}

\section{PARTIE EXPERIMENTALE}

Pour l'étude du système, 22 alliages ont été préparés par une méthode métallurgique classique (Tableau 2) en utilisant des métaux purs. La température a été progressivement élevée à $1000{ }^{\circ} \mathrm{C}$, puis les alliages ont été recuits à $280^{\circ} \mathrm{C}$ pendant 120 jours.

L'identification et la détermination des compositions des alliages ont été réalisées par diffraction des rayons $\mathrm{X}$, microsonde de Castaing, microscopie optique et mesures de la microdureté Vickers. Les structures cristallines et les distances inter-réticulaires ont été tirées de la littérature [2, 3, 13].

Les observations optiques ont été réalisées sur des échantillons polis, puis soumis à une attaque par une solution alcoolique de $\mathrm{HNO}_{3}$. Un appareillage PMT-3 a été utilisé pour les mesures de microdureté $[14,15]$.

\section{RÉSULTATS ET DISCUSSION}

Le tableau 3 contient les résultats détaillés des mesures de microdureté des phases ( $\mathrm{DAg}, \mathrm{HECu} \mathrm{Eu}_{3} \mathrm{Ti}$ type), ](Mg-type)). Les valeurs sont généralement plus basses que celles des phases respectives dans le système Ag-Sn-Zn ( $\mathrm{D}$ fcc solutions solides, les phases Het $\mathrm{H}$ et les phases ]). Les valeurs différent de 2-3 fois pour les phases $D(A g(S n, I n)$ et $A g(S n, Z n)), 20 \%$ pour les phases du type $\mathrm{Mg}$ et $10 \%$ pour les phases du type $\mathrm{ECu}_{3} \mathrm{Ti}$.

Il est intéressant de mentionner qu'une structure lamellaire a été observée dans les échantillons Nos. 7, 9, 15 et 18 qui sont partiellement liquides à la température de travail. Les spécimens Nos. 7, 9 et 18 appartiennent au champ binaire ] $+\mathrm{L}$.

La figure 5 représente l'image MEB d'échantillon No. 9. La région blanche démontre la phase ] $\left(\mathrm{X}_{\mathrm{Ag}}=0.738, \mathrm{X}_{\mathrm{Sn}}=0.178\right.$ et $\left.\mathrm{X}_{\mathrm{In}}=0.084\right)$, la phase gris foncé est le liquide et les dendrites - ], ayant une composition hors d'équilibre $\left(\mathrm{X}_{\mathrm{Ag}}=0.700, \mathrm{X}_{\mathrm{Sn}}=0.132\right.$ et $\left.\mathrm{X}_{\mathrm{In}}=0.164\right)$. Cette composition peut être expliquée par une vitesse de refroidissement très lente ou par une croissance de la phase à température ambiante. Les dendrites ont des angles de 60 et $120^{\circ}$ qui sont typiques pour le réseau hexagonal. 
Tableau 2. Compositions chimiques des échantillons utilisés pour l'étude des équilibres entre phase dans le système Ag-Sn-In. $X_{\mathrm{Ag}}, \mathrm{X}_{\mathrm{Sn}}, \mathrm{X}_{\mathrm{In}}$ sont les fractions molaires, No les numéros d'échantillon.

\begin{tabular}{|c|c|c|c|c|c|c|c|}
\hline No & $\mathrm{X}_{\mathrm{Ag}}$ & $\mathrm{X}_{\mathrm{Sn}}$ & $\mathrm{X}_{\mathrm{In}}$ & $\mathrm{No}$ & $\mathrm{X}_{\mathrm{Ag}}$ & $\mathrm{X}_{\mathrm{Sn}}$ & $\mathrm{X}_{\mathrm{In}}$ \\
\hline \hline 1 & 0,935 & 0,051 & 0,025 & 11 & 0,045 & 0,903 & 0,052 \\
\hline 2 & 0,869 & 0,105 & 0,027 & 12 & 0,066 & 0,522 & 0,412 \\
\hline 3 & 0,784 & 0,175 & 0,042 & 13 & 0,737 & 0,019 & 0,244 \\
\hline 4 & 0,752 & 0,219 & 0,029 & 14 & 0,586 & 0,099 & 0,315 \\
\hline 5 & 0,014 & 0,952 & 0,034 & 15 & 0,647 & 0,100 & 0,253 \\
\hline 6 & 0,816 & 0,075 & 0,109 & 16 & 0,053 & 0,690 & 0,257 \\
\hline 7 & 0,742 & 0,158 & 0,100 & 17 & 0,392 & 0,296 & 0,312 \\
\hline 8 & 0,621 & 0,281 & 0,099 & 18 & 0,206 & 0,694 & 0,100 \\
\hline 9 & 0,497 & 0,402 & 0,100 & 19 & 0,413 & 0,391 & 0,196 \\
\hline 10 & 0,060 & 0,821 & 0,120 & 20 & 0,643 & 0,038 & 0,319 \\
\hline 11 & 0,043 & 0,858 & 0,099 & 21 & 0,319 & 0,034 & 0,647 \\
\hline
\end{tabular}

Tableau 3. Résultats des mesures de microdureté Vickers.

\begin{tabular}{|c|c|l|c|c|c|c|c|}
\hline No. & Phases & $\mathrm{H}[\mathrm{MPa}]$ & $\begin{array}{c}\text { Erreur } \\
{[\mathrm{MPa}]}\end{array}$ & No. & Phases & $\mathrm{H}[\mathrm{MPa}]$ & $\begin{array}{c}\text { Erreur } \\
{[\mathrm{MPa}]}\end{array}$ \\
\hline 1 & $\mathrm{D}$ & 64 & $\mathrm{r} 2$ & 9 & ] & 113 & $\mathrm{r} 1$ \\
\hline 3 & $\mathrm{~J}$ & 118 & $\mathrm{r} 3$ & 13 & ] & 93 & $\mathrm{r} 1$ \\
\hline 4 & $\mathrm{H}$ & 122 & $\mathrm{r} 2$ & 15 & ] & 121 & $\mathrm{r} 3$ \\
\hline 7 & $\mathrm{]}$ & 103 & $\mathrm{r} 1$ & 19 & ] & 99 & $\mathrm{r} 1$ \\
\hline 8 & $\mathrm{~J}$ & 107 & $\mathrm{r} 2$ & & & & \\
\hline
\end{tabular}

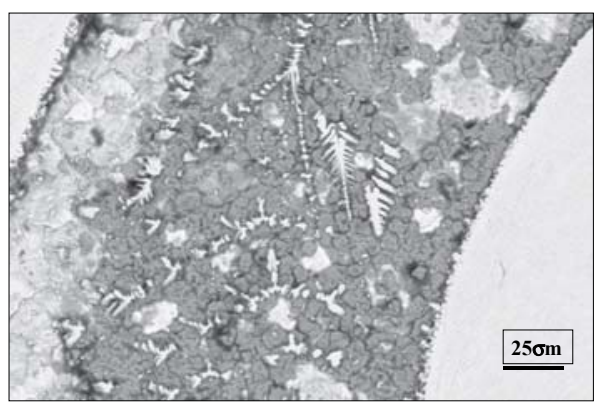

Figure 1. Image MEB de l'échantillon No.9. Les dendrites claires sont identifiées comme étant la phase ], avec une composition hors d'équilibre, la région grise - le liquide et la matrice blanche - la phase ] d'équilibre.

Korhonen et Kivilahti ont cherché à localiser le point eutectique ternaire. Ils ont donné la composition $\mathrm{X}_{\mathrm{Ag}}=0.025, \mathrm{X}_{\mathrm{Sn}}=0.475$ et $\mathrm{X}_{\mathrm{In}}=0.500$ et la température $113^{\circ} \mathrm{C}$. Cette composition est très proche de celles des échantillons Nos. 13 et 17 . L'analyse thermique montre l'existence de pics endothermiques a $113^{\circ} \mathrm{C}$ (Fig.2) pour ces échantillons. Tous ces spécimens, sauf le numéro 23, montrent au moins un pic a haute température.

Le pic à $215^{\circ} \mathrm{C}$ observé dans le spécimen No 27 peut être attribué à la réaction peritéctique

Une autre possibilité est

$$
\mathrm{L}+\mathrm{H} \square(\mathrm{ESn})+\mathrm{J}\left(\mathrm{InSn}_{4}\right)
$$

$$
\mathrm{L}+\left[\square(\mathrm{ESn})+\mathrm{J}\left(\mathrm{InSn}_{4}\right)\right.
$$

En considérant les courbes de refroidissement on a trouvé que pour l'échantillon No. 23, le pic a $113^{\circ} \mathrm{C}$ est bien reproduit. On peut donc conclure que cet alliage est situé très près du point eutectique. 


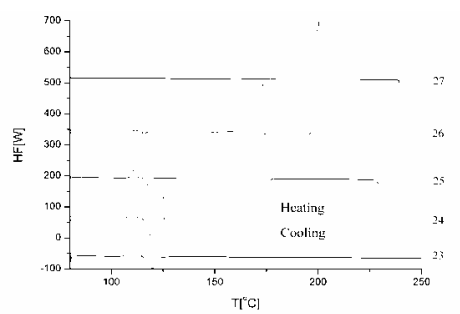

Figure 2. Courbe DSC (échantillons $23-27, v=0.5 \mathrm{~K} / \mathrm{min}$.). Le pic à $113^{\circ} \mathrm{C}$ est associé à la réaction eutectique ternaire.

Les résultats de nos études, corrélés aux données pour les systèmes binaires [1-11] de la littérature nous ont permis de construire la coupe isotherme du système Ag-Sn-In a $280^{\circ} \mathrm{C}$ (Fig.3).

Figure 3. Coupe isotherme du système $\mathrm{Ag}-\mathrm{Sn}-\mathrm{In}$ à $280^{\circ} \mathrm{C}$.

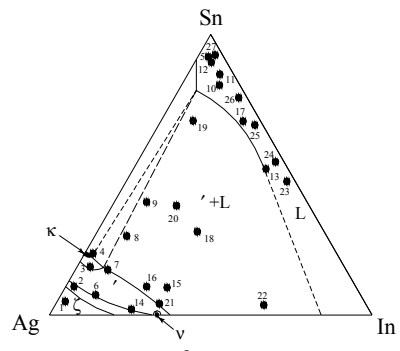

\section{CONCLUSIONS}

Nous avons pu construire la coupe isotherme du système Ag-Sn-In à $280{ }^{\circ} \mathrm{C}$. Les micro-duretés des phases D, Het ] ont été mesurées. Les intervalles d'homogénéité des solutions solides et des phases intermétalliques ont été déterminés.

\section{Références}

[1] F. Hua and J. Glazer, "Lead-free solders for electronic assembly", Design and Reliability of Solders and Solder Interconnections, the Minerals, Metals \&Materials Society, pp. 65-73 (1997).

[2] M. Hansen and K. Anderko, Constitution of Binary Alloys, 2nd edn., McGrow-Hill, NY, 1958, p. 1488.

[3] ASM International, CD ROM, Binary Alloy Phase Diagrams, Second Edition, Metals Park Ohio, 1996.

[4] B. J. Lee, C. S. Oh, J. H. Shim, J. Electronic Mater., 25 (6) (1996) 983.

[5] Y. Xie, Z. Qiao, J. Phase Equilibria, 17(3) (1996) 208.

[6] T.-M. Korhonen, J.K. Kivilahti, J. Electronic Mater., 27 (3) (1998) 149.

[7] J. Inohana, X.J.Liu, I.Ohnuma, R. Kainuma, K. Ishida, Abstracts of the Jpn. Inst. Metals, 125 (1999) 368.

[8] I. Ohnuma, M. Miyashita, X. J. Liu, H. Ohtani, K. Ishida, IEMT/IMC Proceedings, (2000) 104.

[9] H.Ohtani, M. Miyashita, K. Ishida, Abstracts of the Jpn. Inst. Metals, 63 (1999), 685.

[10] I. Ohnuma, X.J. Liu, H.Ohtani and K. Ishida, J. Electronic Mater., 28 (1999) 1164.

[11] I. Ohnuma, X.J. Liu, K. Ishida, R.Kainuma, Y. Cui, H. Ohtani, S. Ishihara, J. Inohana, J. El. Mater., 29 (2000) 10.

[12] Z. Moser, W. Gasior, J. Pstrus, W. Zakulski, I. Ohnuma, X. J. Liu, Y. Inohana and K. Ishida, J. Electronic Mater., 30 (8) (2001) 1120.

[13] JCPDS-ICDD, Powder Diffraction Files, CD-ROM, 1995.

[14] G.P. Vassilev, E.S. Dobrev, S.K. Evtimova, J.-C. Tedenac, JALCOM 327 (2001) 285.

[15] G.P. Vassilev, J.-C. Tedenac, S.K. Evtimova, E.S. Dobrev, JALCOM 334 (2002) 182. 